\title{
Compensação Fuzzy Proporcional-Derivativa de Incertezas no Controle de Vibrações de Treliças com Memória de Forma
}

\author{
Philippe E. Medeiros ${ }^{*, * *}$ Marcelo A. Savi ${ }^{* * *}$ \\ Wallace M. Bessa ${ }^{* * *}$ \\ * Universidade Federal Rural de Pernambuco, Unidade Acadêmica do \\ Cabo de Santo Agostinho, PE, Brasil (e-mail: \\ philippe.medeiros@ufrpe.br). \\ ** Universidade Federal do Rio Grande do Norte, Programa de \\ Pós-Graduação em Engenharia Mecânica, RN, Brasil (e-mail: \\ philippeemedeiros@gmail.com). \\ *** Universidade Federal do Rio de Janeiro, Departamento de \\ Engenharia Mecânica, RJ, Brasil (e-mail: savi@mecanica.ufrj.br). \\ **** Universidade Federal do Rio Grande do Norte, Departamento de \\ Engenharia Mecânica, RN, Brasil (e-mail: wmbessa@ct.ufrn.br).
}

\begin{abstract}
In this work, a sliding mode controller with proportional-derivative fuzzy compensation of uncertainties is applied in the active vibration control of a shape memory alloy two-bar truss. This system has a very rich dynamics and can present chaotic responses depending on the parameters of an external disturbance. In order to design a simpler controller, two constitutive models are considered: a polynomial model for the development of the control law, and another, which presents responses similar to experimental data, to simulate the thermomechanical behavior of the structure elements. In this way, this approach has the advantage of obtaining both simpler and intelligent control law, and allows to evidence its robustness. Numerical simulations are presented in order to demonstrate the effectiveness of the proposed strategy.

Resumo: Neste trabalho, um controlador por modos deslizantes com compensação fuzzy proporcional-derivativa de incertezas é empregado no controle ativo de vibrações de uma treliça de duas barras de liga com memória de forma. Esse sistema possui uma dinâmica bastante rica e complexa, podendo apresentar comportamentos caóticos dependendo dos parâmetros da perturbação externa. No intuito de facilitar o projeto do controlador, dois modelos constitutivos são utilizados: um polinomial, para o desenvolvimento da lei de controle, e um outro mais sofisticado, para simular o comportamento termomecânico dos elementos da estrutura, o qual apresenta resultados compatíveis com experimentos. Dessa forma, esta abordagem tem como vantagem a obtenção de uma lei de controle mais simples, inteligente, e permite que a sua robustez seja evidenciada. Simulações numéricas são apresentadas para comprovar a eficácia da estratégia proposta.
\end{abstract}

Keywords: Sliding mode control; fuzzy logic; shape memory structures; two-bar truss; shape memory alloys.

Palavras-chaves: Controle por modos deslizantes; lógica fuzzy; estruturas com memória de forma; treliça de duas barras; ligas com memória de forma.

\section{INTRODUÇÃO}

A análise de estabilidade de sistemas estruturais é alvo de muitas pesquisas, principalmente devido aos comportamento complexos que eles podem apresentar (Kounadis and Kratzik, 1995). Devido à complexidade associada à estruturas mais bem elaboradas, modelos mais simples, até mesmo com um ou dois graus de liberdade, são geralmente utilizados para estudar os aspectos gerais relacionados ao comportamento dinâmico de estruturas. Um desses mode-

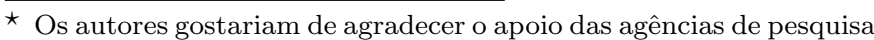
brasileiras CNPq, CAPES e FAPERJ e a PPg/UFRN.
}

los é o da treliça plana de duas barras, também conhecida como treliça de von Mises. A treliça de duas barras é um modelo bastante importante para definir as principais características de estabilidade das estruturas emolduradas, bem como dos arcos e de muitos outros fenômenos físicos associados à flambagem e bifurcações (Bazant and Cedolin, 2010). Uma das principais característica da treliça de duas barras é que, para um dado nível de carregamento, duas configurações de equilíbrio são possíveis. A transição de uma configuração para outra, caracterizada por um comportamento não linear, conhecido como comportamento snap-through, ocorre quando uma carga mecânica externa crescente é aplicada sobre a estrutura (Pecknold et al., 
1985). O comportamento da treliça se torna ainda mais rico quando o material que a compõe apresenta um comportamento não linear, como no caso da estrutura do presente trabalho.

As ligas com memória de forma (LMFs) são assim conhecidas devido ao efeito memória de forma e de superelasticidade apresentadas por elas. O efeito memória de forma é o fenômeno no qual o material consegue recuperar a sua forma após ter se deformado permanentemente, através de um processamento térmico específico. Já a superelasticidade consiste em o material recuperar toda a deformação imposta sobre ele, acompanhado de um laço de histerese em um ciclo de carregamento-descarregamento, desde que a liga se encontre em uma faixa de temperatura específica. Ambos os fenômenos estão associados às transformações martensíticas que ocorrem nas LMFs, devido aos ciclos termomecânicos aos quais elas são submetidas. Essas características as tornam aplicáveis em áreas como: estruturas e materiais compósitos, nas indústrias automotiva e aeroespacial, na fabricação de atuadores e sistemas microeletromecânicos, em robótica, na área biomédica, entre outras (Lagoudas, 2008; Mohd Jani et al., 2014).

Estruturas com elementos de liga com memória de forma são também conhecidas como estruturas inteligentes, já que são capazes de mudar suas formas geométricas ou propriedades físicas com a finalidade de executar alguma tarefa específica. Muitas aplicações em diferentes áreas da ciência e engenharia têm sido desenvolvidas com essas características, através da utilização de materiais inteligentes. É possível encontrar estruturas que possuem como atuadores os seus próprios elementos, como as que são feitas de LMF. Utilizando esse material como atuador, é possível obter boas respostas, sem contar que eles apresentam facilidade de fabricação, são relativamente leves e são capazes de produzir altas cargas de deslocamentos (Benafan et al., 2014). Uma revisão completa sobre o controle de vibrações de estruturas inteligentes é apresentada em Amezquita-Sanchez et al. (2014).

O objetivo do presente trabalho é desenvolver um controlador por modos deslizantes suavizados com compensação fuzzy proporcional-derivativa de incertezas para controlar os níveis de vibração de uma treliça de duas barras de LMF. No intuito de facilitar o projeto do controlador, um modelo polinomial é utilizado no desenvolvimento da lei de controle, enquanto um outro modelo mais sofisticado, capaz inclusive de descrever o comportamento histerético do material, é empregado para simular o comportamento termomecânico dos elementos da estrutura. Tendo em vista que o controlador por modos deslizantes requer a utilização de um modelo para estimar a dinâmica da planta a ser controlada, e que o modelo com histerese, dada sua complexidade, não seria o mais adequado, o modelo polinomial será o empregado na elaboração da lei de controle. Esta abordagem tem como principal vantagem a obtenção de uma lei de controle mais simples e mais robusta.

\section{MODELO DINÂMICO}

A treliça de duas barras é formada por dois elementos idênticos no plano 2D, com mesmo comprimento $L$ e mesma área de seção transversal $A_{s t}$, formando um ângulo

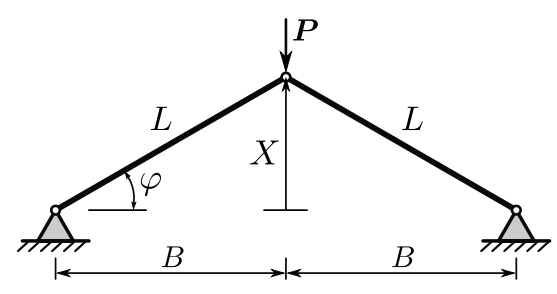

Figura 1. Representação de uma treliça de duas barras.

$\varphi$ com o plano da superfície dos apoios, como ilustrado na Figura 1.

É importante destacar que os elementos podem rotacionar livremente em torno das suas respectivas articulações e que a carga crítica de Euler é considerada suficientemente alta tal que o fenômeno da flambagem não ocorra. A massa da estrutura $(m)$ é considerada como concentrada no ponto de junção das duas barras, e apenas movimentos verticais e simétricos são considerados. Apesar da simplificação, a resposta apresentada ainda é capaz de exibir comportamentos complexos de grande importância para a análise de estruturas.

Aplicando a segunda lei de Newton na junção das barras, chega-se à seguinte equação de movimento:

$$
-2 F \operatorname{sen} \varphi-c \dot{X}+P=m \ddot{X}
$$

onde $F$ é a força em cada barra, $P$ é uma força externa aplicada verticalmente na junção das barras, $c$ é o coeficiente de amortecimento viscoso equivalente da estrutura e $X$ o movimento vertical simétrico do ponto central.

Um modelo matemático desenvolvido por Paiva et al. (2005) é empregado para descrever o comportamento termomecânico da LMF da treliça do presente trabalho. Este modelo é capaz de descrever o comportamento termomecânico geral das LMFs, apresentando uma boa concordância com dados experimentais. Nesse modelo é considerado a tensão $(\sigma)$, a deformação $(\varepsilon)$, a temperatura $(T)$ e três variáveis de estado, dadas por $\rho_{1}, \rho_{2}$ e $\rho_{3}$, que estão relacionadas com as frações volumétricas associadas à cada fase da liga, sendo $\rho_{1}$ a fração associada à martensita não maclada devido a tração $(M+), \rho_{2}$ a fração da martensita não maclada devido à compressão $(M-)$ e $\rho_{3}$ a fração associada à austenita $(A)$. Uma quarta fase $\left(\rho_{4}\right)$ é ainda considerada, que é a fração da martensita maclada $(M)$, e pode ser obtida da condição de coexistência das fases, ou seja: $\rho_{4}=1-\left(\rho_{1}+\rho_{2}+\rho_{3}\right)$. Com essas considerações, o comportamento termomecânico da LMF da estrutura pode ser descrito por:

$$
\begin{aligned}
\sigma= & E \varepsilon+\left[\alpha+E \alpha_{h}\right]\left(\rho_{2}-\rho_{1}\right)-\kappa\left(T-T_{0}\right), \\
\dot{\rho}_{1}= & \bar{\eta}_{1}^{-1}\left\{\alpha \varepsilon+\Lambda+\left[2 \alpha_{h} \alpha+E \alpha_{h}^{2}\right]\left(\rho_{2}-\rho_{1}\right)+\right. \\
& \left.+\alpha_{h}\left[E \varepsilon-\kappa\left(T-T_{0}\right)\right]-\partial_{1} J_{\pi}\right\}+\partial_{1} J_{\chi}, \\
\dot{\rho}_{2}= & \bar{\eta}_{2}^{-1}\left\{-\alpha \varepsilon+\Lambda-\left[2 \alpha_{h} \alpha+E \alpha_{h}^{2}\right]\left(\rho_{2}-\rho_{1}\right)+\right. \\
& \left.-\alpha_{h}\left[E \varepsilon-\kappa\left(T-T_{0}\right)\right]-\partial_{2} J_{\pi}\right\}+\partial_{2} J_{\chi}, \\
\dot{\rho}_{3}= & \bar{\eta}_{3}^{-1}\left\{0,5\left(E_{M}-E_{A}\right)\left[\varepsilon+\alpha_{h}\left(\rho_{2}-\rho_{1}\right)\right]^{2}+\right. \\
& +\left(\kappa_{A}-\kappa_{M}\right)\left(T-T_{0}\right)\left[\varepsilon+\alpha_{h}\left(\rho_{2}-\rho_{1}\right)\right]+ \\
& \left.+\Lambda_{3}-\partial_{3} J_{\pi}\right\}+\partial_{3} J_{\chi},
\end{aligned}
$$


onde $E$ é o módulo de elasticidade e $\kappa$ está associado ao coeficiente de expansão térmica da liga, sendo definidos, respectivamente, por: $E=E_{M}+\rho_{3}\left(E_{A}-E_{M}\right)$ e $\kappa=$ $\kappa_{M}+\rho_{3}\left(\kappa_{A}-\kappa_{M}\right)$, onde os índices " $A$ " e " $M$ ", que aparecem em $\kappa$ e $E$, estão associados as fases autenítica e martesítica, respectivamente. O parâmetro $\alpha_{h}$ é utilizado para definir o comprimento horizontal do laço de histerese do gráfico tensão-deformação do material, enquanto $\alpha$ ajuda no controle da sua dimensão vertical. Os parâmetros $\bar{\eta}_{i}(i=1,2,3)$ estão associados às dissipações relacionadas as transformações de fase, enquanto $\Lambda=\Lambda(T)$ e $\Lambda_{3}=$ $\Lambda_{3}(T)$ estão associados aos níveis de tensão para o início das transformações. O conjunto de restrições, bem como os parâmetros $\Lambda$ e $\Lambda_{3}$, podem ser consultados em Paiva et al. (2005).

Os termos $\partial_{i} J_{\pi}(i=1,2,3)$ são subdiferenciais da função indicatriz $J_{\pi}$ em relação a $\rho_{i}$, e a função indicatriz $J_{\pi}=J_{\pi}\left(\rho_{1}, \rho_{2}, \rho_{3}\right)$ está relacionada ao conjunto convexo $\pi$, que fornece as restrições internas relacionadas a coexistência das fases presentes na liga (Rockafellar, 1970). Já $\partial_{i} J_{\chi}(i=1,2,3)$ são subdiferenciais da função indicatriz $J_{\chi}\left(\dot{\varepsilon}, \dot{T}, \dot{\rho}_{1}, \dot{\rho}_{2}, \dot{\rho}_{3}\right)$ em relação a $\dot{\rho}_{i}$, e a função indicatriz $J_{\chi}$ está relacionada ao conjunto convexo $\chi$, que fornece restrições associadas à evolução das transformações de fase (Rockafellar, 1970).

A deformação para cada barra da estrutura pode ser definida da seguinte maneira:

$$
\varepsilon=\frac{L}{L_{0}}-1=\frac{\cos \varphi_{0}}{\cos \varphi}-1,
$$

sendo $L_{0}$ e $\varphi_{0}$ os valores nominais de $L$ e $\varphi$, respectivamente. Logo, sabendo que $F=\sigma A_{s t}$, e substituindo as equações $(2-6)$ na equação $(1)$, define-se a seguinte equação de movimento para o ponto central da estrutura:

$$
\begin{array}{r}
m \ddot{X}+c \dot{X}+2 A_{s t} \frac{X}{\left(X^{2}+B^{2}\right)^{1 / 2}}\left\{E \left[\frac{\left(X^{2}+B^{2}\right)^{1 / 2}}{L_{0}}+\right.\right. \\
\left.-1]+\left[\alpha+E \alpha_{h}\right]\left(\rho_{2}-\rho_{1}\right)-\kappa\left(T-T_{0}\right)\right\}=P(t) .
\end{array}
$$

Considerando uma excitação periódica sobre a estrutura, $P(t)=P_{0} \operatorname{sen}(\omega t)$, a equação de movimento $(7)$ pode ser reescrita de maneira adimensional como:

$$
\begin{aligned}
\dot{x}= & y, \\
\dot{y}= & -\bar{\xi} y-\zeta_{E}\left[1-\frac{1}{\left(x^{2}+\beta^{2}\right)^{1 / 2}}\right] x+ \\
& -\left[\left(\bar{\alpha}+\zeta_{E} \alpha_{h}\right)\left(\rho_{2}-\rho_{1}\right)+\right. \\
& \left.-\bar{\kappa} \zeta_{\kappa}\left(\theta-\theta_{0}\right)\right] \frac{x}{\left(x^{2}+\beta^{2}\right)^{1 / 2}}+\gamma \operatorname{sen}(\Omega \tau),
\end{aligned}
$$

onde os parâmetros adimensionais de (8) são definidos da seguinte maneira:

$$
\begin{aligned}
& x=\frac{X}{L_{0}}, \beta=\frac{B}{L_{0}}, \theta=\frac{T}{T_{M}}, \tau=\omega_{0} t, \dot{x}=\frac{d x}{d \tau} \\
& \Omega=\frac{\omega}{\omega_{0}}, \zeta_{E}=\frac{E}{E_{M}}, \zeta_{\kappa}=\frac{\kappa}{\kappa_{M}}, \bar{\xi}=\frac{c}{m \omega_{0}}, \bar{\alpha}=\frac{\alpha}{E_{M}}, \\
& \gamma=\frac{P_{0}}{m L_{0} \omega_{0}^{2}}, \omega_{0}^{2}=\frac{2 E_{M} A_{s t}}{m L_{0}} \text { e } \bar{\kappa}=\frac{\kappa_{M} T_{M}}{E_{M}} .
\end{aligned}
$$

\section{PROJETO DO CONTROLADOR}

Nesta seção, um controlador por modos deslizantes suavizados com compensação fuzzy proporcional-derivativa é aplicado a treliça de duas barras de liga com memória de forma, para assegurar que o vetor de estados $\mathbf{x}=\left[\begin{array}{ll}x & y\end{array}\right]^{\top}$ atinja o vetor de estados desejados $\mathbf{x}_{d}=\left[\begin{array}{ll}x_{d} & y_{d}\end{array}\right]^{\top}$ e o erro de estabilização $\tilde{\mathbf{x}}=\mathbf{x}-\mathbf{x}_{d}=\left[\begin{array}{ll}\tilde{x} & \tilde{y}\end{array}\right]^{\top} \rightarrow \mathbf{0}$ quando $\tau \rightarrow \infty$, mesmo na presença de incertezas e de uma perturbação externa. Para descrever o comportamento termomecânico do material da estrutura, o projeto do controlador é desenvolvido a partir do modelo polinomial proposto por Falk (1980), enquanto um outro modelo, o qual apresenta resultados compatíveis com experimentos (Paiva et al., 2005), é utilizado para descrever o comportamento termomecânica do material da estrutura a ser controlada. Com o auxílio do compensador fuzzy, verifica-se que essa abordagem é possível (além de mais simples), uma vez que ele consegue compensar toda incerteza presente no sistema, tanto incertezas paramétricas como dinâmicas não modeladas, além de perturbações externas.

Para que a ação de controle seja efetivada e consiga atenuar os níveis de vibrações da estrutura, será considerado um atuador na junção das duas barras, como mostra a Figura 2 , de atuação linear.

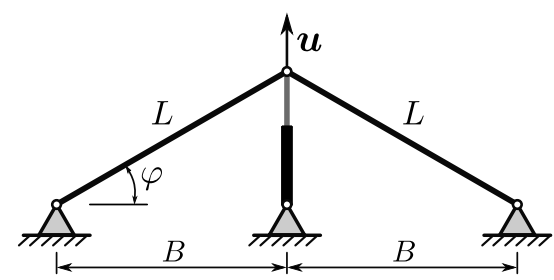

Figura 2. Representação esquemática da treliça de duas barras com o atuador.

Após a adição do atuador externo ao ponto central da estrutura, o sistema dado em (8) será representado da seguinte maneira:

$$
\begin{aligned}
& \dot{x}=y, \\
& \dot{y}=d+f+u,
\end{aligned}
$$

onde $u$ é a ação de controle, $d=\gamma \operatorname{sen}(\Omega \tau)$ é a perturbação externa e $f$ representa a dinâmica do sistema, a qual é descrita pela dinâmica do sistema da equação (8).

No projeto do controlador foram adotadas as seguintes hipóteses:

Hipótese 1. O vetor de estados x está disponível.

Hipótese 2. A dinâmica do sistema $f$ não é conhecida, porém é limitada por uma função de x. Logo, temos: $|\hat{f}-f| \leq F(\mathbf{x})$, onde $\hat{f}$ é a estimativa de $f$.

Hipótese 3. A perturbação externa $d$ não é conhecida, porém é limitada por $D$, ou seja $|d| \leq D$.

A técnica de controle por modos deslizantes tem como objetivo principal converter um problema de rastreamento de trajetória de ordem $n$ em $\mathbf{x}$, em um problema de estabilização de ordem $n-1$ em $s(\mathbf{x})$, sendo $s: \mathbb{R}^{n} \rightarrow \mathbb{R}$ uma superfície de deslizamento, definida pela equação 
escalar $s=\left(\frac{d}{d t}+\lambda\right)^{n-1} \tilde{x}=0$, para um sistema dinâmico de ordem $n$ (Slotine and Li, 1991). Considerando a superfície de deslizamento como $s=\tilde{y}+\lambda \tilde{x}$, o controlador por modos deslizante com compensação fuzzy proporcional-derivativa para a treliça de duas barras pode ser definido através da combinação de um controle equivalente $u=\dot{y}_{d}-\hat{f}-$ $\hat{d}(\tilde{x}, \tilde{y})-\lambda \tilde{y}$ com outro termo, $-K \operatorname{sat}(s / \phi)$, para conferir robustez ao sistema, da seguinte maneira:

$$
u=\dot{y}_{d}-\hat{f}-\hat{d}(\tilde{x}, \tilde{y})-\lambda \tilde{y}-K \operatorname{sat}(s / \phi),
$$

onde $\hat{d}$ é a estimativa de $d, \hat{f}$ é a estimativa de $f$ e $\operatorname{sat}(s / \phi)$ é função saturação, definida como:

$$
\operatorname{sat}(s / \phi)=\left\{\begin{array}{cc}
\operatorname{sgn}(s / \phi) & \text { se }|s / \phi| \geq 1, \\
s / \phi & \text { se }|s / \phi|<1,
\end{array}\right.
$$

sendo sgn a função sinal e $\phi$ a espessura da camada limite da superfície de deslizamento.

Nessa análise, a perturbação externa $d$ não será levada em conta na equação da lei de controle (11), porém ela será estimada pelo sistema de compensação fuzzy, que também compensará as demais incertezas da dinâmica do sistema adotada no controlador, a qual é representada por $\hat{f}$.

Tendo em vista que o controlador por modos deslizantes requer a utilização de um modelo para estimar a dinâmica da planta a ser controlada, e que o modelo com histerese, dada sua complexidade, não seria o mais adequado, um modelo polinomial (Falk, 1980) será empregado para descrever o comportamento termomecânico do material para o controlador. Esse polinômio pode ser representado em função da deformação uniaxial do material, como: $\sigma=a_{1}\left(T-T_{M}\right) \varepsilon-a_{2} \varepsilon^{3}+a_{3} \varepsilon^{5}$, sendo $a_{1}, a_{2}$ e $a_{3}$ parâmetros associados ao material. Então, substituindo $\sigma$ em (1), a dinâmica da estrutura com o modelo polinomial pode ser descrita, de maneira adimensional, como:

$$
\begin{aligned}
\hat{f}= & -\xi x_{2}+x_{1}\left\{-\left[(\theta-1)-3 \alpha_{2}+5 \alpha_{3}\right]+\right. \\
& +\left[(\theta-1)-\alpha_{2}+\alpha_{3}\right]\left(x_{1}^{2}+\beta^{2}\right)^{-1 / 2}+ \\
& -\left[3 \alpha_{2}-10 \alpha_{3}\right]\left(x_{1}^{2}+\beta^{2}\right)^{1 / 2}+ \\
& +\left[\alpha_{2}-10 \alpha_{3}\right]\left(x_{1}^{2}+\beta^{2}\right)+5 \alpha_{3}\left(x_{1}^{2}+\beta^{2}\right)^{3 / 2}+ \\
& \left.-\alpha_{3}\left(x_{1}^{2}+\beta^{2}\right)^{2}\right\},
\end{aligned}
$$

onde $\xi$ é um parâmetro adimensional relacionado ao coeficiente de amortecimento equivalente da estrutura, e $\alpha_{2}$ e $\alpha_{3}$ são parâmetros adimensionais associados as constantes $a_{2}$ e $a_{3}$ do modelo polinomial (Savi et al., 2002). Os demais parâmetros de (13), estão definidos em (9). Para garantir que a superfície de deslizamento seja atrativa $\forall \mathbf{x} \in \mathbb{R}^{n}, K$ deve ser definido como:

$$
K \geq \eta+F+D+|\hat{d}|
$$

A fim de obter uma boa aproximação para a perturbação externa e para as incertezas do sistema, a estimativa $\hat{d}$ é calculada diretamente por um algoritmo fuzzy proporcional-derivativo. De acordo com Kosko (1994), os sistemas fuzzy podem ser considerados como aproximadores universais e, portanto, podem aproximar qualquer função em um conjunto compacto a uma precisão arbitrária. O sistema de inferência adotado foi o TSK de ordem zero (Takagi-Sugeno-Kang), cujas regras podem ser declaradas de maneira linguística como:

$$
\text { Se z é } \mathcal{Z}_{r} \text {, então } \hat{d}_{r}=\hat{D}_{r} ; r=1,2, \ldots, k \text {. }
$$

onde $\mathcal{Z}_{r}$ são conjuntos fuzzy, cujas funções de associação podem ser escolhidas adequadamente, e $\hat{D}_{r}$ é o valor de saída de cada uma das $k$ regras fuzzy.

Para o compensador proporcional-derivativo, uma função $\hat{d}: \mathbb{R}^{2} \rightarrow \mathbb{R}$ deve ser definida com base em dois universos de discurso, de modo que $\hat{d} \approx f(\tilde{x}, \tilde{y})$, para $\tilde{x} \in \mathcal{X}$ e $\tilde{y} \in \mathcal{Y}$, sendo $\mathcal{X}$ e $\mathcal{Y}$ os universos de discurso do erro e da derivada do erro. É importante destacar que os universos de discurso $\mathcal{X}$ e $\mathcal{Y}$ são divididos, respectivamente, em $n$ e $m$ conjuntos fuzzy. Para calcular $\hat{d}$, a T-norma produto deve ser aplicada para criar um vetor de dimensão $n \times m$ que associa cada função de pertinência do primeiro universo de discurso $\mu_{i}: \mathbb{R} \rightarrow \mathbb{R}$, para $i=1,2, \ldots, n$, com cada função de pertinência do segundo universo de discurso $\mu_{j}: \mathbb{R} \rightarrow \mathbb{R}$, para $j=1,2, \ldots, m$, ou seja:

$$
\mu_{i \times j}=\mu_{i}(\tilde{x}) \mu_{j}(\tilde{y}) .
$$

Como temos dois universos de discurso, o vetor associado às saídas de cada função, ou vetor de base de regras, deve ter a mesma dimensão do vetor criado em (15). O universo de discurso é divido de forma que só são ativados no máximo dois conjuntos fuzzy por vez, então os vetores $\mu_{i}$ e $\mu_{j}$ só têm dois elementos não nulos cada. Se os dois vetores apresentarem norma unitária, pode-se afirmar que o vetor resultante da T-norma produto conservará a norma unitária. Desta forma, baseado no método TSK de ordem zero, o compensador fuzzy será dado por:

$$
\hat{d}(\tilde{x}, \tilde{y})=\sum_{r=1}^{r=n \times m} D_{r} \mu_{r}(\tilde{x}, \tilde{y}),
$$

No presente trabalho o método TSK de ordem zero é utilizado por questão de simplicidade e por ser suficiente para compensar, de forma satisfatória, as incertezas do sistema analisado.

Para um melhor entendimento do esquema de controle adotado, a Figura 3 ilustra o diagrama de blocos da lei de controle (11).

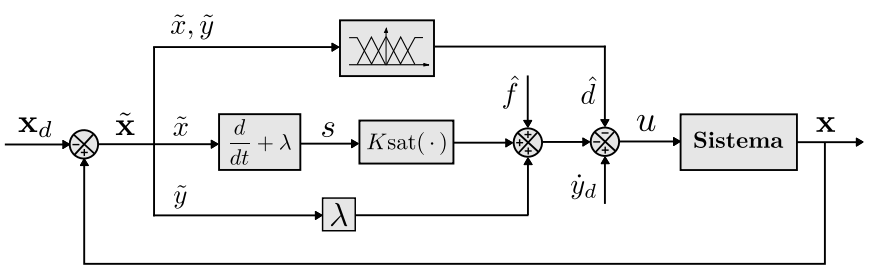

Figura 3. Diagrama de blocos da lei de controle adotada.

\section{SIMULAÇÕES NUMÉRICAS}

Para realizar as simulações numéricas do modelo com histerese, foi utilizado o método de Runge-Kutta de quarta ordem, com um passo de integração de $\Delta \tau=\pi / 1000 \Omega$. Para o cálculo de $\rho_{i}$, as equações $(3-5)$ são resolvidas de forma desacoplada, não levando em consideração as subdiferenciais, tanto da função indicatriz $J_{\pi}$ como de $J_{\chi}$. Se os resultados obtidos para $\rho_{i}$ não satisfizerem as restrições impostas pelo conjunto convexo $\pi$, um algoritmo de projeção ortogonal calcula um valor otimizado de $\rho_{i}$, que são determinados a cada passo de integração. Em todas as simulações, foram considerados os parâmetros 
que estão presentes na Tabela 1. Os valores escolhidos representam um típico comportamento de uma LMF a uma temperatura de $373 \mathrm{~K}$.

Tabela 1. Propriedades do material utilizadas na simulação do modelo com histerese.

\begin{tabular}{|c|c|c|c|}
\hline $\begin{array}{c}E_{A}(\mathrm{GPa}) \\
56\end{array}$ & $\begin{array}{c}E_{M}(\mathrm{GPa}) \\
56\end{array}$ & $\begin{array}{c}\alpha(\mathrm{MPa}) \\
140\end{array}$ & $\begin{array}{c}\alpha_{h} \\
0,053\end{array}$ \\
\hline$l(\mathrm{MPa})$ & $l_{0}(\mathrm{MPa})$ & $l_{0}^{A}(\mathrm{MPa})$ & $l^{A}(\mathrm{MPa})$ \\
\hline 43,5 & 0,2 & 0,62 & 175 \\
\hline $\begin{array}{c}\kappa_{A}\left(\mathrm{MPaK}^{-1}\right) \\
0,75\end{array}$ & $\begin{array}{c}\kappa_{M}\left(\mathrm{MPaK}^{-1}\right) \\
0,16\end{array}$ & $\begin{array}{c}T_{M}(\mathrm{~K}) \\
291,4\end{array}$ & $\begin{array}{c}T_{A}(\mathrm{~K}) \\
307,7\end{array}$ \\
\hline 0,75 & $\begin{array}{c}\bar{\eta}^{C} \text { (MPa s) } \\
10,5\end{array}$ & $\begin{array}{c}\bar{\eta}^{D}(\mathrm{MPa} \mathrm{s}) \\
28\end{array}$ & \\
\hline
\end{tabular}

Savi et al. (2002) e Savi and Nogueira (2010) estudaram treliças de duas barras feitas com ligas com memória de forma, e apresentaram resultados numéricos do seu comportamento dinâmico considerando diferentes modelos para descrever o comportamento termomecânica do material. Savi et al. (2002) utilizaram o modelo polinomial proposto por Falk (1980) e Savi and Nogueira (2010) utilizaram o modelo proposto em Paiva et al. (2005). Em ambas as análises foi possível observar tanto respostas caóticas como periódicas. As respostas caóticas foram observadas através de diagramas de bifurcação, os quais apresentaram regiões com nuvens de pontos em algumas faixas de $\gamma$ e $\Omega$. O comportamento dinâmico desse sistema pode ser visto detalhadamente em Savi et al. (2002) e Savi and Nogueira (2010).

Uma resposta caótica desse sistema, obtida através da equação (8), pode ser observada na Figura 4, através do seu plano de fase para $\gamma=0,011$ e $\Omega=0,375$. A Figura 4 está associada a um típico atrator estranho, o qual está relacionado a uma das respostas caóticas do sistema. Observe que a trajetória ocupa todo o espaço de fase, visitando todos os pontos de equilíbrio.

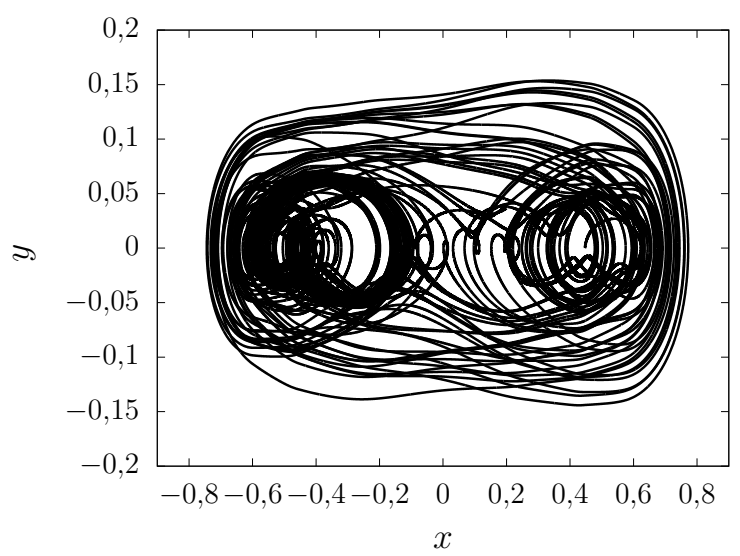

Figura 4. Plano de fase do sistema para $\gamma=0,011 \mathrm{e}$ $\Omega=0,375$.

Com relação ao controlador e a estabilização do sistema, os universos de discurso foram definidos tendo como base a região do erro residual de estabilização, que pode ser definida em função dos parâmetros $\phi$ e $\lambda$ (Bessa, 2009). Dessa forma, foram considerados os seguintes universos de discurso: $\mathcal{X}=\{-\phi / \lambda \leq \tilde{x} \leq \phi / \lambda\}$ e $\mathcal{Y}=\{-2 \phi \leq$ $\tilde{y} \leq 2 \phi\}$, com funções de pertinência trapezoidais nas extremidades, e triangulares no centro. Com relação aos centros das funções de pertinência, foi adotada uma maior concentração de seus valores próxima a zero, para que o ganho da saída do controlador diminua nessa região e o sistema atinja a resposta desejada com o menor erro possível. Nas simulações numéricas, adotou-se como estado desejado $\mathbf{x}_{d}=\left[\begin{array}{ll}0,53 & 0,0\end{array}\right]^{\top}$, com a condição inicial $\mathbf{x}(0)=$ $\left[\begin{array}{ll}0,447 & 0,0\end{array}\right]^{\top}$, e os seguintes parâmetros adimensionais: $\theta=1,28, \bar{\xi}=0,0$ e $\beta=0,894$.

As Figuras 5 e 6 mostram a estabilização do sistema, e o seu respectivo esforço de controle, provenientes do controle por modos deslizantes suavizados com compensação fuzzy PD projetado. Para os centros das funções de pertinência, foram adotados os valores de $c_{\tilde{x}}=\{-\phi / \lambda ;-0,08 \phi / \lambda ;-0,03 \phi / \lambda ; 0,03 \phi / \lambda ; 0,08 \phi / \lambda ; \phi / \lambda\}$ $\times 10^{-1}$ e $c_{\tilde{y}}=\{-2,0 \phi ;-1,0 \phi ;-0,02 \phi ; 0,02 \phi ; 1,0 \phi ; 2,0 \phi\} \times$ $10^{-1}$, para $\mathcal{X}$ e $\mathcal{Y}$, respectivamente. Os valores para cada regra são atribuídos heuristicamente.

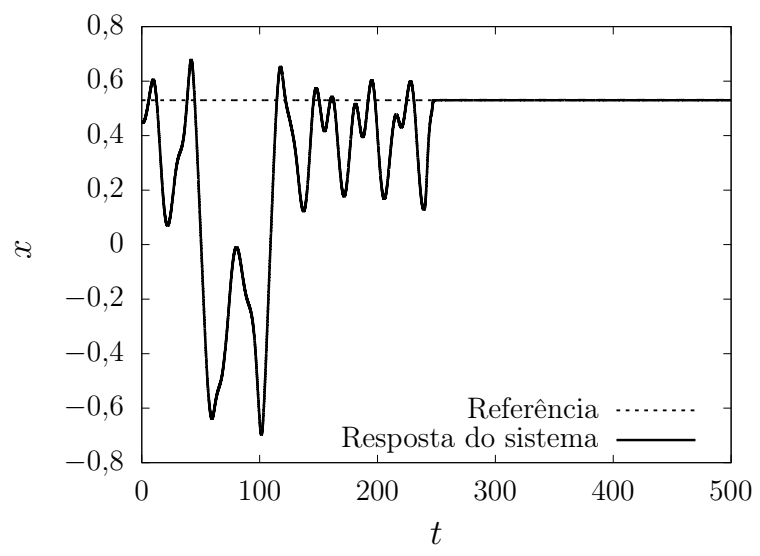

Figura 5. Estabilização do sistema em $x_{d}=0,53$ para $\gamma=0,011$ e $\Omega=0,375$.

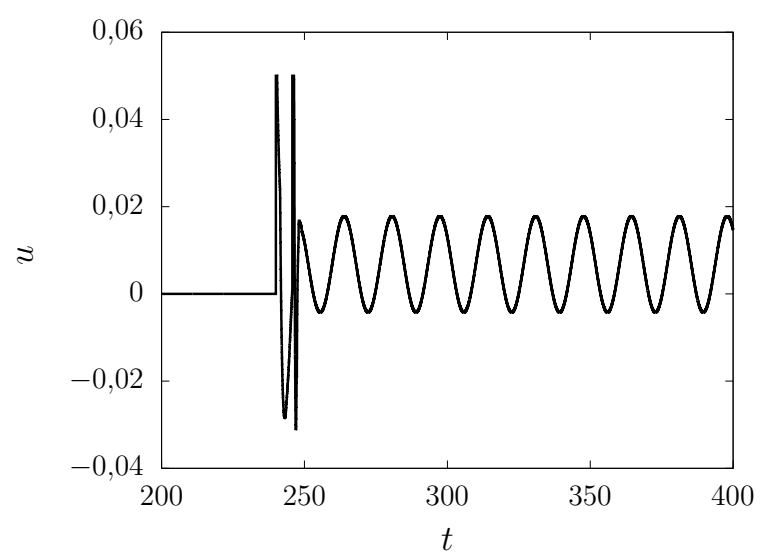

Figura 6. Esforço de controle.

Podemos observar das Figuras 5 e 6 que a estratégia de controle adotada reduz de forma bastante satisfatória os níveis de vibração da estrutura, e evita, dessa forma, o comportamento snap-through, que é bastante prejudicial ao sistema. Também é possível notar que quando o controlador é ativado $(\tau=240)$, o movimento caótico 
é substituído por um suave e muito próximo ao estado desejado.

Na Figura 6, pode-se visualizar o momento em que a compensação fuzzy é acionada, através das variações bruscas que ocorrem no esforço de controle próximo a $\tau=240$. Através da análise comparativa mostrada na Figura 7, pode-se facilmente observar a melhora na performance do controlador proposto com a compensação fuzzy em relação ao controlador utilizando somente a técnica de controle por modos deslizantes. A compensação fuzzy proporcionalderivativa garante uma convergência mais suave do sistema para o estado desejado em relação a uma compensação fuzzy proporcional pura. Por considerar o erro e sua derivada, o sistema tem a capacidade de dosar a compensação para o controlador. Por exemplo, se o erro é positivo, mas a sua derivada é negativa, isso indica que o sistema irá naturalmente convergir para a redução do erro. No caso da configuração apenas proporcional ao erro, ocorreria um aumento da compensação, e isso faria com que o sistema ultrapassasse o ponto desejado no regime transitório da resposta.

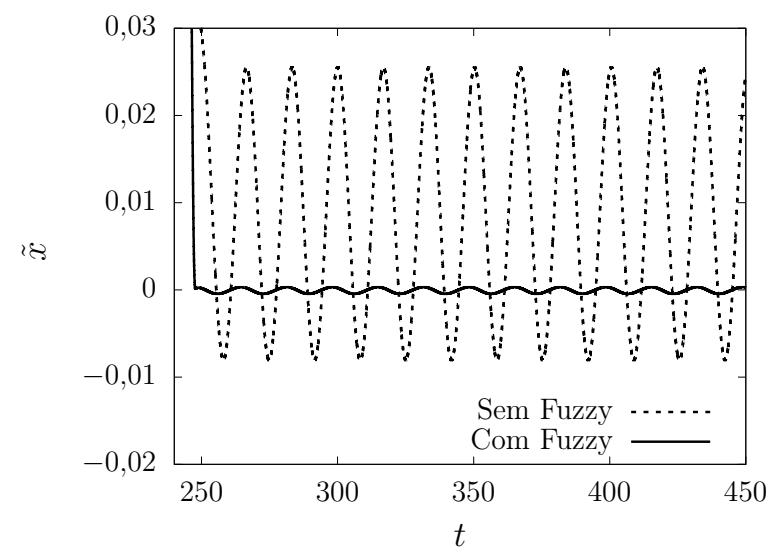

Figura 7. Análise comparativa do erro com e sem o compensador fuzzy no controlador.

\section{CONCLUSÃO}

Neste artigo, um controlador por modos deslizantes com compensação fuzzy proporcional-derivativa é considerado para atenuar vibrações em uma treliça de duas barras com memória de forma. Assume-se um modelo que apresenta resultados semelhantes com experimentos para descrever o comportamento termomecânico das barras e um modelo polinomial para descrever esse mesmo comportamento para o controlador. Esse sistema apresenta um comportamento complexo, podendo exibir respostas caóticas ou periódicas dependendo dos parâmetros da perturbação externa. Simulações numéricas mostram a robustez do controlador proposto frente as incertezas presentes no modelo e a presença de perturbações externas. O desempenho aprimorado sobre o controlador por modos deslizantes convencional também é demonstrado. Deve-se destacar que a robustez do controlador para imprecisões de modelagem é uma questão importante, pois permite o uso de um modelo simples para fins de controle de sistemas com comportamentos dinâmicos mais complexos.

\section{AGRADECIMENTOS}

Os autores gostariam de agradecer o apoio das agências de pesquisa brasileiras CNPq, CAPES e FAPERJ, e a PróReitoria de Pós-Graduação da UFRN.

\section{REFERÊNCIAS}

Amezquita-Sanchez, J.P., Dominguez-Gonzalez, A., Sedaghati, R., de Jesus Romero-Troncoso, R., and Osornio-Rios, R.A. (2014). Vibration control on smart civil structures: A review. Mechanics of Advanced $M a-$ terials and Structures, 21(1), 23-38.

Bazant, Z.P. and Cedolin, L. (2010). Stability of Structures. World Scientific Publishing Co. Pte. Ltd.

Benafan, O., Brown, J., Calkins, F., Kumar, P., Stebner, A., Turner, T., Vaidyanathan, R., Webster, J., and Young, M. (2014). Shape memory alloy actuator design: Casmart collaborative best practices and case studies. International Journal of Mechanics and Materials in Design, 10(1), 1-42.

Bessa, W.M. (2009). Some remarks on the boundedness and convergence properties of smooth sliding mode controllers. International Journal of Automation and Computing, 6(2), 154-158.

Falk, F. (1980). Model free energy, mechanics, and thermodynamics of shape memory alloys. Acta Metallurgica, 28(12), 1773-1780.

Kosko, B. (1994). Fuzzy systems as universal approximators. IEEE Transactions on Computers, 11(43), 13291333.

Kounadis, A. and Kratzik, W. (1995). Nonlinear Stability of Structures. Springer.

Lagoudas, D.C. (2008). Shape Memory Alloys: Modeling and Engineering Applications.

Mohd Jani, J., Leary, M., Subic, A., and Gibson, M.A. (2014). A review of shape memory alloy research, applications and opportunities. Materials and Design, 56, 1078-1113.

Paiva, A., Savi, M.A., Braga, A.M.B., and Pacheco, P.M.C.L. (2005). A constitutive model for shape memory alloys considering tensile-compressive asymmetry and plasticity. International Journal of Solids and Structures, 42(11-12), 3439-3457.

Pecknold, D., Ghaboussi, J., and Healey, T. (1985). Snapthrough and bifurcation in a simple structure. Journal of Engineering Mechanics, 111(7), 909-922.

Rockafellar, R.T. (1970). Convex Analysis. Princeton University Press.

Savi, M.A. and Nogueira, J.B. (2010). Nonlinear dynamics and chaos in a pseudoelastic two-bar truss. Smart Materials and Structures, 19(11), 115022.

Savi, M.A., Pacheco, P.M.C.L., and Braga, A.M.B. (2002). Chaos in a shape memory two-bar truss. International Journal of Non-Linear Mechanics, 37(8), 1387-1392.

Slotine, J.J.E. and Li, W. (1991). Applied Nonlinear Control. Prentice Hall. 